Article

\title{
Bioassays against Pinewood Nematode: Assessment of a Suitable Dilution Agent and Screening for Bioactive Essential Oils
}

\author{
Pedro Barbosa ${ }^{1}$, Jorge M. S. Faria ${ }^{2}$, Marta D. Mendes ${ }^{2}$, Luís Silva Dias ${ }^{3}$, Maria Teresa Tinoco ${ }^{4}$, \\ José G. Barroso $^{2}$, Luis G. Pedro ${ }^{2}$, Ana Cristina Figueiredo ${ }^{2}$ and Manuel Mota ${ }^{1, *}$
}

1 NemaLab, ICAAM - Instituto de Ciências Agrárias e Ambientais Mediterrânicas", Universidade de Évora, Núcleo da Mitra, Ap. 94, 7002-554 Évora, Portugal

2 Universidade de Lisboa, Faculdade de Ciências de Lisboa, DBV, IBB, Centro de Biotecnologia Vegetal, C2, Campo Grande, 1749-016 Lisboa, Portugal

3 Departamento de Biologia, Universidade de Évora, 7002-554 Évora, Portugal

4 ICAAM, Departamento de Química, Universidade de Évora, 7000-671 Évora, Portugal

* Author to whom correspondence should be addressed; E-Mail: mmota@uevora.pt; Tel.: +35-12-6676-0881; Fax: +35-12-6676-0914.

Received: 21 September 2012; in revised form: 10 October 2012 / Accepted: 10 October 2012 / Published: 19 October 2012

\begin{abstract}
Acetone was investigated and found to be an appropriate alternative to Triton $\mathrm{X}-100$ as a solvent of essential oils in bioassays aimed to investigate their effects on pinewood nematode (Bursaphelenchus xylophilus) mortality. Therefore it was used as dilution agent to screen the effectiveness of fifty two essential oils against this pest. Thirteen essential oils were highly effective, resulting in more than $90 \%$ pinewood nematode mortality at $2 \mathrm{mg} / \mathrm{mL}$, with six of them resulting in $100 \%$ mortality. $\mathrm{LC}_{100}$ values ranged between $0.50 \mathrm{mg} / \mathrm{mL}$ and $0.83 \mathrm{mg} / \mathrm{mL}$ for the essential oils of Origanum vulgare and Satureja montana, respectively. Essential oils were submitted to gas chromatography and gas chromatography-mass spectrometry analysis and their chemical composition established. Data from essential oils with $100 \%$ mortality at $2 \mathrm{mg} / \mathrm{mL}$ and other essential oils previously found to have $\mathrm{LC}_{100} \leq 2 \mathrm{mg} / \mathrm{mL}$ was combined, their chemical profiles investigated by correspondences analysis plus automatic classification.
\end{abstract}

Keywords: Bursaphelenchus xylophilus; essential oils; nematicide activity; acetone; Triton X-100 


\section{Introduction}

The pinewood nematode (PWN), Bursaphelenchus xylophilus L., is a serious threat to forest ecosystems at a global scale, affecting wood trade and its industry [1]. Capable of completely destroying Pinus spp. trees, the nematode was classified as an A2 type quarantine pest by the European Plant Protection Organization. In 1999, the nematode was detected for the first time in Portugal, expanding the distribution in the Northern hemisphere (United States, Canada, Mexico, China, Japan, South Korea and Taiwan). More recently, new outbreaks were reported on Madeira Island [2] and in Spain [3]. These developments alerted the EU and new restrictions along with new disease control measures were implemented [4].

Many of the most effective chemicals used for controlling PWN are toxic, expensive or lead to accumulation in the soil, causing significant environmental impacts. In face of the recent EU environmental restrictions, it became necessary to develop environmentally safer control techniques based on natural products. Essential oils have long been known to have significant biological activities. Recent studies have shown that some essential oils appear to have good nematicidal activity against the PWN under laboratory conditions [5-8].

Triton X-100 is a nonionic detergent-type surfactant, known for its capacity to solubilize membrane proteins. This wetting agent is commonly used for dilution purposes due to the fact it increases the penetrating and spreading properties of liquids. However, our accumulated experience shows that the use of Triton X-100 might be inappropriate for routine use because of the difficulty in obtaining homogenous dissolution of essential oils.

Acetone is a polar aprotic solvent commonly employed for all purpose laboratory uses, particularly given its miscibility with water. Acetone has been rated as a Generally Recognized As Safe (GRAS) substance when present in beverages, baked foods, desserts, and preserves at concentrations ranging from 5 to $8 \mathrm{mg} / \mathrm{L}$ [9].

The search for bioactive phytochemicals relies heavily on screening a large number of plant sources followed by bioassay guided fractionation of the most promising ones. Therefore, the present study was set out to: (a) compare the suitability of Triton X-100 and acetone as solvents for plant essential oils to control PWN; (b) widen the screening of species that might be sources of phytochemicals able to completely control PWN.

\section{Results and Discussion}

\subsection{Assessment of Triton X-100 and Acetone Nematicidal Activity}

Mean PWN mortality using Triton X-100 (50 $\mu \mathrm{g} / \mathrm{mL})$ or acetone $(1 \% \mathrm{v}: \mathrm{v})$ was $2.26 \pm 0.26 \%$ and $1.53 \pm 0.19 \%$, respectively, and no significant difference $(p=0.027)$ was found between them. Therefore, and despite the fact that sometimes acetone has been used to control plant parasitic nematodes [10], its use as a solvent for essential oils in bioassays does not raise concerns regarding PWN mortality. 


\subsection{Comparative Evaluation of Essential Oils' Nematicidal Activity Using Triton X-100 or Acetone as Solvent}

Worldwide research in this area employs some kind of detergent (usually Triton X-100) to dilute oils. Plant essential oils have been routinely prepared by serial dilution with distilled water containing Triton X-100 and tested on B. xylophilus [6,8,11-14]. Triton-X is recognized as a good dilution agent for essential oils, able to increase tissue permeability, relatively easy to handle and, because it is not volatile, it allows concentrations to remain essentially constant over time. However, macroscopic examination clearly shows that some essential oils are difficult to dissolve in Triton X-100. One or more oil drops can easily remain inside the detergent and the same can occur when new dilutions are prepared. Ultrasound or temperature increases are commonly used to break up these oils drops. In the present study, when submitting oil/Triton solution to ultrasound irradiation the oil drops remained after $20 \mathrm{~min}$ and only started to disappear after $30 \mathrm{~min}$, probably because of a simultaneous temperature increase over time. On the other hand, warming the solution is not feasible given the high volatility of the essential oils. This observation prompted us to check for an alternative solvent. Essential oils of Cymbopogon citratus, Origanum vulgare and Satureja montana previously found to result in more than 90\% PWN mortality [8] were tested using acetone as dilution agent and the results compared with those previously found using Triton X-100.

Whenever significant differences were found, the use of Triton X-100 always resulted in reduced PWN mortality compared with acetone. In addition, the variability of effects was always higher using Triton X-100 (Table 1). These results suggest that Triton X-100 may be less effective than acetone in providing a homogeneous solution of essential oils when a dilution series is prepared from a higher concentration, especially in the case of $O$. vulgare. Acetone thus seemed better suited for essential oil dilution in nematicide bioassays, than Triton X-100.

Table 1. Bursaphelenchus xylophilus mortality (mean \pm SE, in percentage) when significant differences were found between the use of Triton X-100 and acetone as dilution agents of essential oils $(p \leq 0.006)$.

\begin{tabular}{|c|c|c|c|c|}
\hline Species & Dilution agent & $0.25 \mathrm{mg} / \mathrm{mL}$ & $0.5 \mathrm{mg} / \mathrm{mL}$ & $1 \mathrm{mg} / \mathrm{mL}$ \\
\hline \multirow[t]{2}{*}{ Cymbopogon citratus } & Triton X-100* & $14.98 \pm 2.17$ & $81.60 \pm 1.72$ & - \\
\hline & Acetone & $83.80 \pm 1.08$ & $89.39 \pm 1.18$ & - \\
\hline \multirow[t]{2}{*}{ Origanum vulgare } & Triton X-100* & $2.78 \pm 0.68$ & $3.72 \pm 0.56$ & $26.61 \pm 3.83$ \\
\hline & Acetone & $94.90 \pm 1.06$ & $98.81 \pm 0.51$ & $100.00 \pm 0.00$ \\
\hline \multirow[t]{2}{*}{ Satureja montana 1} & Triton X-100* & $7.13 \pm 1.19$ & - & - \\
\hline & Acetone & $57.60 \pm 2.44$ & - & - \\
\hline
\end{tabular}

* data from Barbosa et al. [8]; - no significant differences between Triton X-100 and acetone treatments $(p>0.01)$.

However, the concentrations tested have no biological meaning per se and more important than comparing essential oils effects at defined and more or less arbitrary concentrations is the comparison of biological meaningful parameters derived from the overall response of PWN mortality to a gradient of essential oils concentrations, namely the minimum concentration of essential oil effective against PWN $(l)$, the symmetry of the distribution of PWN mortality $(c)$ and the minimum concentration of essential oil resulting in $100 \%$ PWN mortality $\left(\mathrm{LC}_{100}\right)$. 
With $C$. citratus essential oil no significant differences between Triton X-100 and acetone were found in $l(p=0.090)$, in $c(p=0.107)$, in the maximum mortality $(p=0.040)$ and in the essential oil concentration at which maximum mortality would occur $(p=0.015)$.

Conversely, with $O$. vulgare essential oil, significant differences were found in $l(p=0.001)$, $c\left(p=2.8 \times 10^{-8}\right)$ and $\operatorname{LC}_{100}\left(p=2.7 \times 10^{-11}\right)$. As might be expected from the individual concentration comparisons, $\mathrm{LC}_{100}$ values were much higher when Triton X-100 was used $(1.984 \pm 0.008 \mathrm{mg} / \mathrm{mL})$ than with acetone $(0.498 \pm 0.028 \mathrm{mg} / \mathrm{mL})$. Previously, using Triton X-100 [8], O. vulgare was the only source of essential oil responsible for negative asymmetry in PWN mortality (mean $c$ value $4.797 \pm 0.109)$ while all the other essential oils resulted in positive asymmetry. Using acetone, all essential oils resulting in $100 \%$ PWN mortality at $2 \mathrm{mg} / \mathrm{mL}$ also showed positive asymmetry (Table 2), meaning that interaction of factors occurred, possibly acting multiplicatively [15].

Table 2. Estimated values (mean $\pm \mathrm{SE}$ ) of highest concentrations of essential oil at which the mortality is strictly zero $(l)$, symmetry of mortality distribution $(c)$, and concentrations necessary to result in $100 \%$ Bursaphelenchus xylophilus mortality $\left(\mathrm{LC}_{100}\right)$.

\begin{tabular}{lccc}
\hline Species & $\boldsymbol{l}$ & $\boldsymbol{c}$ & $\mathbf{L C}_{\mathbf{1 0 0}}$ \\
\hline Origanum vulgare $*$ & $0.100 \pm 0.0017 \mathrm{a}$ & $1.770 \pm 0.095 \mathrm{a}$ & $0.498 \pm 0.028 \mathrm{a}$ \\
Ruta graveolens 1 & $0.096 \pm 0.0014 \mathrm{a}$ & $1.799 \pm 0.052 \mathrm{a}$ & $0.571 \pm 0.046 \mathrm{~b}$ \\
Ruta graveolens 2 & $0.095 \pm 0.0008 \mathrm{a}$ & $1.915 \pm 0.135 \mathrm{a}$ & $0.663 \pm 0.032 \mathrm{c}$ \\
Satureja montana $1 *$ & $0.099 \pm 0.0003 \mathrm{a}$ & $1.946 \pm 0.008 \mathrm{a}$ & $0.793 \pm 0.002 \mathrm{~d}$ \\
Satureja montana 2 & $0.089 \pm 0.0024 \mathrm{~b}$ & $2.832 \pm 0.056 \mathrm{~b}$ & $0.819 \pm 0.007 \mathrm{~d}$ \\
Satureja montana 3 & $0.089 \pm 0.0013 \mathrm{c}$ & $2.798 \pm 0.020 \mathrm{c}$ & $0.828 \pm 0.001 \mathrm{~d}$ \\
\hline
\end{tabular}

* Essential oils also tested in Barbosa et al. [8] using Triton X-100. Acetone used as solvent. All concentrations in $\mathrm{mg} / \mathrm{mL}$. In each column, means with the same letter do not differ for an experiment-wise error rate of 0.01 .

This discrepancy of $O$. vulgare is surprising and hard to explain given the high similarity between its chemical profile and those of the most part of the other essential oils (Figure 1 and discussion below), but it completely disappears when acetone is used, thereby supporting that using acetone as solvent might be a better choice than using Triton X-100. Finally, with $S$. montana essential oil, no significant differences were found in $l(p=0.012)$ and $\operatorname{LC}_{100}(p=0.352)$, while c differed significantly $(p=0.004)$ between Triton X-100 (2.310 \pm 0.064$)$ and acetone $(1.946 \pm 0.008)$, with the latter making more clear the positive asymmetry of PWN mortality distribution.

Macroscopic inspection showed clear solutions without oil drops, revealing that essential oils were completely and homogeneously dissolved. The only downside of this solvent may be its volatility, causing concentration fluctuations in the stock solutions, particularly if kept for long periods. This problem can be diminished if the stock solution is kept at $-20{ }^{\circ} \mathrm{C}$ until use.

Overall, Triton X-100 replacement by acetone is clearly a sound choice for all practical reasons. Results of PWN response to essential oils using acetone do not differ from results using Triton X-100 or, when they do, acetone based results are more consistent. To our knowledge, despite having been used in the trunk injection technique to control PWN [16], this is the first report on the use of acetone as solvent for essential oil dilution in PWN nematicide bioassays. 
Figure 1. Ordination of essential oils (circles) and constituents (squares) in the first two factors of correspondences analysis.

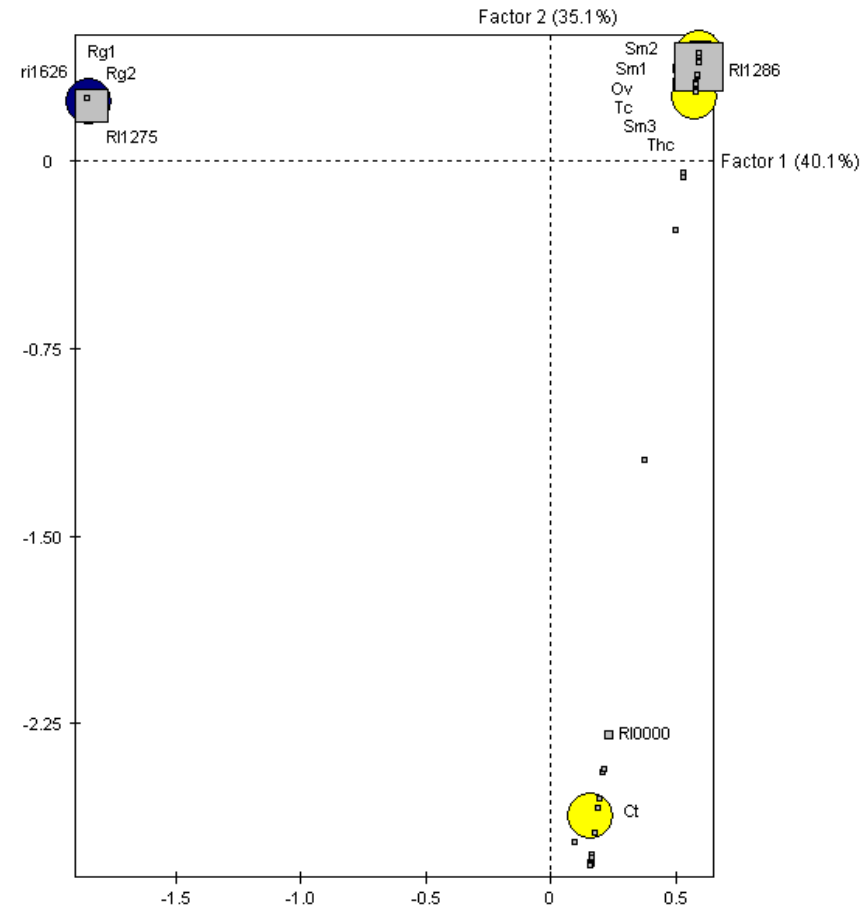

Sizes are proportional to weights. In blue, essential oils from class 1 , in yellow from class 2 . $\mathrm{Ct}$ Chamaespartium tridentatum, Ov Origanum vulgare, Rg1 Ruta graveolens 1, Rg2 Ruta graveolens 2, Sm1 Satureja montana 1, Sm2 Satureja montana 2, Sm3 Satureja montana 3, Tc Thymbra capitata, The Thymus caespititius. RI0000 other unidentified compounds, RI1275 2-undecanone, RI1286 carvacrol, ri1626 unidentified compounds in the essential oil of Ruta graveolens. Data for C. tridentatum, O. vulgare, S. montana 1, T. capitata and T. caespititius from Barbosa et al. [8].

\subsection{Essential Oils Nematicide Activity}

Fifty two essential oils isolated from 29 taxa were screened for PWN nematicide activity using acetone as dilution agent (Table 3). As detailed in the next section, for several species, more than one essential oil chemotype, or essential oils isolated from different plant parts of the same species, were assessed.

With 38 of the tested oils, the $99 \%$ confidence interval of mean mortality at $2 \mathrm{mg} / \mathrm{mL}$ using acetone did not include zero mortality and therefore significant effects of essential oils on PWN occurred (Table 3). However, full mortality was reached with only six oils from three different species, belonging to the Lamiaceae (O. vulgare and S. montana) and Rutaceae (Ruta graveolens).

In general, the response of PWN to different essential oil chemotypes or essential oils isolated from diverse plant parts from the same species varied little or not at all. However PWN mortality in response to Thymus caespititius strongly varied with the oils bioassayed, ranging between $6.06 \pm 0.62 \%$ and $99.44 \pm 0.26 \%$ but never attaining $100 \%$ in all replicates as found previously with an oil from a different population [8]. 
Table 3. Nematicidal activity of 52 essential oils against Bursaphelenchus xylophilus exposed for $24 \mathrm{~h}$ to a $2 \mathrm{mg} / \mathrm{mL}$ solution.

\begin{tabular}{|c|c|c|c|c|c|c|c|}
\hline Code & Family / Species & $\begin{array}{l}\text { Collection place } \\
\text { or source }^{b}\end{array}$ & Date & $\begin{array}{c}\text { Plant part }^{\mathrm{c}} \\
\text { Status }\end{array}$ & I.P. $^{\mathrm{d}}$ & $\begin{array}{l}1 \text { yield } \\
0, v / w)\end{array}$ & Mortality $^{\mathrm{e}}(\mathbf{\%})$ \\
\hline \multirow[t]{2}{*}{$\mathrm{Scm}$} & Schinus molle L. & Évora & 2005 & Leaves, Fresh & $\mathrm{H}$ & 0.40 & $1.54 \pm 0.47$ \\
\hline & Apiaceae & & & & & & \\
\hline$A l$ & $\begin{array}{l}\text { Angelica lignescens } \\
\text { Reduron et Danton }\end{array}$ & Flores (Az) & 2008 & AP (V), Fresh & $\mathrm{H}$ & 0.08 & $1.75 \pm 0.47$ \\
\hline Cha & $\begin{array}{l}\text { Chaerophyllum azoricum } \\
\text { Trelease }\end{array}$ & Flores (Az) & 2008 & AP (V), Fresh & $\mathrm{H}$ & 0.25 & $1.20 \pm 0.48$ \\
\hline$F v 1$ & Foeniculum vulgare Miller & Graciosa $(\mathrm{Az})$ & 2008 & AP (F), Fresh & $\mathrm{H}$ & 0.33 & $6.21 \pm 0.71 *$ \\
\hline$F v 2$ & Foeniculum vulgare Miller & HS & 2008 & Seeds, Dried & $\mathrm{H}$ & 5.61 & $8.60 \pm 0.81 *$ \\
\hline$F v 3$ & Foeniculum vulgare Miller & HS & 2008 & Seeds, Dried & $\mathrm{H}$ & 6.09 & $9.89 \pm 1.71 *$ \\
\hline$F v 4$ & Foeniculum vulgare Miller & HS & 2008 & Seeds, Dried & $\mathrm{H}$ & 5.88 & $6.29 \pm 0.91 *$ \\
\hline$F v 5$ & Foeniculum vulgare Miller & BPGV & 2008 & Seeds, Dried & $\mathrm{H}$ & 4.78 & $5.95 \pm 0.79 *$ \\
\hline \multirow[t]{2}{*}{$F v 6$} & Foeniculum vulgare Miller & HS & 2008 & Seeds, Dried & $\mathrm{H}$ & 1.07 & $7.13 \pm 0.50 *$ \\
\hline & Cupressaceae & & & & & & \\
\hline$C j$ & $\begin{array}{l}\text { Cryptomeria japonica } \\
\text { (L. fil.) D. Don. }{ }^{\mathrm{f}}\end{array}$ & Flores (Az) & 2008 & Berries, Fresh & $\mathrm{H}$ & 0.41 & $0.79 \pm 0.39$ \\
\hline$J b 1$ & $\begin{array}{l}\text { Juniperus brevifolia (Seub.) } \\
\text { Antoine }\end{array}$ & Flores (Az) & 2008 & Berries, Fresh & $\mathrm{H}$ & 0.06 & $0.84 \pm 0.16 *$ \\
\hline \multirow[t]{2}{*}{$J b 2$} & $\begin{array}{l}\text { Juniperus brevifolia (Seub.) } \\
\text { Antoine }\end{array}$ & Flores (Az) & 2008 & $\mathrm{AP}(\mathrm{V})$, Fresh & $\mathrm{H}$ & 0.45 & $2.56 \pm 0.66$ \\
\hline & Geraniaceae & & & & & & \\
\hline \multirow[t]{2}{*}{$P g 1$} & $\begin{array}{l}\text { Pelargonium graveolens } \\
\text { L’Hér. }\end{array}$ & Lisboa & 2009 & AP (V), Fresh & $\mathrm{H}$ & 0.19 & $74.79 \pm 2.56 *$ \\
\hline & Lamiaceae & & & & & & \\
\hline Mo & Melissa officinalis L. & HS & 2009 & AP (F), Fresh & $\mathrm{H}$ & 0.04 & $99.30 \pm 0.54 *$ \\
\hline$M a$ & Mentha aquatica $\mathrm{L}$. & HS & 2009 & AP (F), Dried & $\mathrm{H}$ & 0.90 & $7.77 \pm 0.83 *$ \\
\hline$M c 1$ & Mentha cervina $\mathrm{L}$. & Beja & 2005 & AP (F), Fresh & $\mathrm{H}$ & 2.00 & $93.56 \pm 1.07 *$ \\
\hline$M c 2$ & Mentha cervina $\mathrm{L}$. & HS & 2009 & AP $(V)$, Dried & $\mathrm{H}$ & 2.12 & $92.57 \pm 1.48 *$ \\
\hline Ms & Mentha spicata $\mathrm{L}$. & Beja & 2009 & AP (V), Fresh & $\mathrm{H}$ & 0.25 & $47.36 \pm 2.22 *$ \\
\hline$N c$ & Nepeta cataria $\mathrm{L}$. & HS & 2009 & AP (F), Fresh & $\mathrm{H}$ & 0.18 & $22.03 \pm 2.66 *$ \\
\hline$O v$ & Origanum vulgare $\mathrm{L} .{ }^{\mathrm{a}}$ & Évora & 2007 & AP (F), Fresh & $\mathrm{H}$ & 1.70 & $100.00 \pm 0.00 *$ \\
\hline$R o 1$ & Rosmarinus officinalis L. & HS & 2009 & Leaves, Dried & $\mathrm{H}$ & 1.95 & $2.55 \pm 0.84$ \\
\hline Ro2 & Rosmarinus officinalis L. & Lisboa & 2009 & AP (V), Fresh & $\mathrm{H}$ & 0.64 & $0.40 \pm 0.41$ \\
\hline Ro3 & Rosmarinus officinalis L. & Lisboa & 2009 & AP (F), Fresh & $\mathrm{H}$ & 1.14 & $2.30 \pm 0.52$ \\
\hline So 1 & Salvia officinalis L. & Lisboa & 2009 & AP (V), Fresh & $\mathrm{H}$ & 0.54 & $1.06 \pm 0.40$ \\
\hline So2 & Salvia officinalis L. & Lisboa & 2009 & $\mathrm{AP}(\mathrm{V})$, Fresh & $\mathrm{H}$ & 0.71 & $0.07 \pm 0.43$ \\
\hline $\operatorname{Sm} 1$ & Satureja montana L. $^{a}$ & HS & 2008 & Leaves, Dried & $\mathrm{H}$ & 1.60 & $100.00 \pm 0.00 *$ \\
\hline $\operatorname{Sm} 2$ & Satureja montana L. & HS & 2009 & AP $(V)$, Dried & $\mathrm{H}$ & 0.55 & $100.00 \pm 0.00 *$ \\
\hline $\operatorname{Sm} 3$ & Satureja montana L. & Beja & 2009 & AP (F), Fresh & $\mathrm{D}-\mathrm{E}$ & - & $100.00 \pm 0.00 *$ \\
\hline$T c 1$ & Thymus caespititius Brot. & Madeira & 2006 & AP (F), Fresh & D-E & - & $6.06 \pm 0.62 *$ \\
\hline$T c 2$ & Thymus caespititius Brot. & S. Jorge (Az) & 2007 & AP (F), Fresh & D-E & - & $97.01 \pm 0.98 *$ \\
\hline
\end{tabular}


Table 3. Cont.

\begin{tabular}{|c|c|c|c|c|c|c|c|}
\hline Code & Family / Species & $\begin{array}{l}\text { Collection place } \\
\text { or source }^{\text {b }}\end{array}$ & Date & $\begin{array}{l}\text { Plant part }^{c} \\
\text { Status }\end{array}$ & I.P. $^{d}$ & $\begin{array}{l}\text { Oil yield } \\
(\%, v / w)\end{array}$ & Mortality $^{\mathrm{e}}(\mathbf{\%})$ \\
\hline$T c 3$ & Thymus caespititius Brot. & Flores (Az) & 2008 & AP (F), Fresh & $\mathrm{H}$ & 0.06 & $94.63 \pm 1.30 *$ \\
\hline$T c 4$ & Thymus caespititius Brot. & Corvo (Az) & 2008 & AP (F), Fresh & $\mathrm{H}$ & 0.22 & $99.44 \pm 0.26 *$ \\
\hline$T c 5$ & Thymus caespititius Brot. & Gerês & 2008 & AP (F), Fresh & $\mathrm{H}$ & 0.35 & $51.61 \pm 3.60 *$ \\
\hline Tc6 & Thymus caespititius Brot. & Graciosa (Az) & 2008 & AP (F), Fresh & $\mathrm{H}$ & 0.38 & $58.21 \pm 2.19 *$ \\
\hline$T c a$ & $\begin{array}{l}\text { Thymus camphoratus } \\
\text { Hoffmans. \& Link }\end{array}$ & Faro & 2008 & AP (F), Fresh & $\mathrm{H}$ & 0.21 & $3.30 \pm 0.59 *$ \\
\hline$T v l$ & $\begin{array}{l}\text { Thymus villosus ssp. } \\
\text { lusitanicus (Boiss.) } \\
\text { Coutinho }\end{array}$ & Leiria & 2008 & AP (F), Fresh & $\mathrm{H}$ & 1.25 & $66.85 \pm 3.44 *$ \\
\hline \multirow[t]{2}{*}{$T z s$} & $\begin{array}{l}\text { Thymus zygis ssp. sylvestris } \\
\text { (Hoffmans. \& Link) } \\
\text { Coutinho }\end{array}$ & Leiria & 2008 & AP (F), Fresh & $\mathrm{H}$ & 0.23 & $24.25 \pm 3.18$ * \\
\hline & Lauraceae & & & & & & \\
\hline$C c$ & $\begin{array}{l}\text { Cinnamomum camphora } \\
\text { (L.) T. Nees \& C.H. Eberm. }\end{array}$ & Coimbra & 2009 & $\begin{array}{l}\text { Branches without } \\
\text { leaves, Dried }\end{array}$ & $\mathrm{H}$ & 0.55 & $1.56 \pm 0.16 *$ \\
\hline$L a$ & $\begin{array}{l}\text { Laurus azorica (Seub.) } \\
\text { J. Franco }\end{array}$ & Flores (Az) & 2008 & AP (V), Fresh & $\mathrm{H}$ & 0.25 & $2.17 \pm 0.66$ \\
\hline Lnc1 1 & $\begin{array}{l}\text { Laurus novocanariensis } \\
\text { Rivas Mart., Lousã, Fern. } \\
\text { Prieto, E. Díaz, J.C. Costa } \\
\text { \& C. Aguiar }\end{array}$ & $\begin{array}{l}\text { Porto da Cruz, } \\
\text { Madeira }\end{array}$ & 2009 & Branches, Fresh & $\mathrm{H}$ & 0.42 & $2.22 \pm 0.39 *$ \\
\hline $\operatorname{Lnc2}$ & $\begin{array}{l}\text { Laurus novocanariensis } \\
\text { Rivas Mart., Lousã, Fern. } \\
\text { Prieto, E. Díaz, J.C. Costa } \\
\text { \& C. Aguiar }\end{array}$ & $\begin{array}{l}\text { Porto da Cruz, } \\
\text { Madeira }\end{array}$ & 2009 & Branches, Fresh & $\mathrm{H}$ & 0.48 & $2.80 \pm 0.34 *$ \\
\hline Lnc3 & $\begin{array}{l}\text { Laurus novocanariensis } \\
\text { Rivas Mart., Lousã, Fern. } \\
\text { Prieto, E. Díaz, J.C. Costa } \\
\text { \& C. Aguiar }\end{array}$ & $\begin{array}{l}\text { Ribeiro Frio, } \\
\text { Madeira }\end{array}$ & 2009 & Branches, Fresh & $\mathrm{H}$ & 0.39 & $2.66 \pm 0.75$ \\
\hline Lnc4 & $\begin{array}{l}\text { Laurus novocanariensis } \\
\text { Rivas Mart., Lousã, Fern. } \\
\text { Prieto, E. Díaz, J.C. Costa } \\
\text { \& C. Aguiar }\end{array}$ & $\begin{array}{l}\text { Ribeiro Frio, } \\
\text { Madeira }\end{array}$ & 2009 & Branches, Fresh & $\mathrm{H}$ & 0.64 & $2.91 \pm 0.44 *$ \\
\hline \multirow[t]{2}{*}{$\operatorname{Lnc5}$} & $\begin{array}{l}\text { Laurus novocanariensis } \\
\text { Rivas Mart., Lousã, Fern. } \\
\text { Prieto, E. Díaz, J.C. Costa } \\
\text { \& C. Aguiar }\end{array}$ & $\begin{array}{l}\text { S. Vicente, } \\
\text { Madeira }\end{array}$ & 2000 & Leaves, Fresh & $\mathrm{H}$ & 0.30 & $4.46 \pm 0.54 *$ \\
\hline & Myrtaceae & & & & & & \\
\hline \multirow[t]{2}{*}{$E g$} & Eucalyptus globulus Labill. & Lisbon & 2009 & AP $(\mathrm{F})$, Fresh & $\mathrm{H}$ & 2.15 & $4.14 \pm 0.85 *$ \\
\hline & & & & & & & \\
\hline$P u 1$ & $\begin{array}{l}\text { Pittosporum undulatum } \\
\text { Vent. }\end{array}$ & Graciosa $(\mathrm{Az})$ & 2008 & Berries, Fresh & $\mathrm{H}$ & 0.21 & $1.22 \pm 0.34$ \\
\hline Pu2 & $\begin{array}{l}\text { Pittosporum undulatum } \\
\text { Vent. }\end{array}$ & Graciosa $(\mathrm{Az})$ & 2008 & Leaves, Fresh & $\mathrm{H}$ & 0.08 & $1.46 \pm 0.44$ \\
\hline
\end{tabular}


Table 3. Cont.

\begin{tabular}{|c|c|c|c|c|c|c|c|}
\hline Code & Family / Species & $\begin{array}{l}\text { Collection place } \\
\text { or source }^{b}\end{array}$ & Date & $\begin{array}{l}\text { Plant part }^{c} \\
\text { Status }\end{array}$ & I.P. ${ }^{\circ}$ & $\begin{array}{l}\text { Oil yield } \\
(\%, v / w)\end{array}$ & $\operatorname{Mortality}^{\mathrm{e}}(\%)$ \\
\hline & Poaceae & & & & & & \\
\hline \multirow[t]{3}{*}{$C y c$} & Cymbopogon citratus (DC) & Faro & 2008 & Leaves, Fresh & $\mathrm{H}$ & 0.80 & $98.86 \pm 0.32 *$ \\
\hline & Stapf. ${ }^{\text {a }}$ & & & & & & \\
\hline & Rutaceae & & & & & & \\
\hline $\mathrm{Ca}$ & Citrus aurantium $\mathrm{L}$. & Évora & 2009 & Leaves, Fresh & $\mathrm{H}$ & 0.31 & $26.59 \pm 1.47 *$ \\
\hline $\operatorname{Rg} 1$ & Ruta graveolens $\mathrm{L}$. & Évora & 2009 & AP $(V)$, Fresh & $\mathrm{H}$ & 2.60 & $100.00 \pm 0.00 *$ \\
\hline \multirow[t]{2}{*}{$\underline{R g 2}$} & Ruta graveolens $\mathrm{L}$. & HS & 2009 & AP (F), Dried & $\mathrm{H}$ & 0.90 & $100.00 \pm 0.00 *$ \\
\hline & Verbenaceae & & & & & & \\
\hline$L c$ & Lippia citriodora Kunth & HS & 2009 & AP $(V)$, Dried & $\mathrm{H}$ & 0.19 & $54.63 \pm 3.53 *$ \\
\hline \multicolumn{8}{|c|}{$\begin{array}{l}\text { Acetone always used as solvent. }{ }^{\text {a }} \text { Essential oils also tested in Barbosa } \text { et al. }[8] \text { using Triton X-100; } \\
{ }^{\mathrm{b}} \mathrm{Az}=\text { Açores; HS }=\text { Herbal shop; BPGV = Banco Português de Germoplasma Vegetal; } \\
{ }^{\mathrm{c}} \mathrm{AP}=\text { aerial part; }(\mathrm{V})=\text { in vegetative phase; }(\mathrm{F})=\text { in flowering phase; }{ }^{\mathrm{d}} \text { I.P. }=\text { isolation procedure; } \\
\text { isolation was either by hydrodistillation }(\mathrm{H}) \text { or distillation-extraction }(\mathrm{D}-\mathrm{E}){ }^{\mathrm{e}} \text { Mortality values with } \\
* \text { have } 99 \% \text { confidence intervals not including zero; }{ }^{\mathrm{f}} \text { Nowadays included in Cupressaceae, } \\
\text { previously Taxodiaceae. }\end{array}$} \\
\hline
\end{tabular}

The three term Weibull function [17] could always be fitted to the effects of the six essential oils able to produce $100 \%$ PWN mortality at $2 \mathrm{mg} / \mathrm{mL}$. Coefficients of determination ranged between 0.525 and $0.996(0.868 \pm 0.030)$. Estimated values of Weibull coefficients $l$ and $c$ and of $\mathrm{LC}_{100}$ are summarized in Table 2, together with significant differences among essential oils for an experiment-wise error rate of 0.01 .

Essential oils from $S$. montana 2 and 3 were the most active at low dosages given their mean values of $l$. All the remaining essential oils had significantly higher mean values of $l$, but absolute differences were relatively small, implying that the minimum active concentration could not provide the rationale for the choice of essentials oils deserving deeper study. In addition, $l$ values intrinsically fail to identify essential oils with the ability to kill $100 \%$ of PWN.

By the contrary, $\mathrm{LC}_{100}$ values make clear that the essential oil from $O$. vulgare $(0.498 \pm 0.028 \mathrm{mg} / \mathrm{mL})$ is a promising source for PWN effective control followed by $R$. graveolens 1 $(0.571 \pm 0.046 \mathrm{mg} / \mathrm{mL})$ and $R$. graveolens $2(0.663 \pm 0.032 \mathrm{mg} / \mathrm{mL})$.

Essential oils for which interactions of effects can be anticipated are better choices for finding one, or at most a few chemicals, able to kill PWN at the lowest possible concentrations [8]. According to this reasoning, essential oils resulting in strongly asymmetric distributions of PWN mortality are preferable. S. montana 2 and 3 have $c$ values relatively close to 3.25 , the lower limit of $c$ for a symmetric distribution. Conversely, the remaining essential oils had $c$ values indicating a highly positive asymmetry of PWN distribution of mortality, strongly suggesting that multiplicative interactions of effects occurred.

In short, essential oil from O. vulgare closely followed by those from R. graveolens 1 and 2 appear as promising sources of phytochemicals worth being selected for bioassay-guided search of highly active compounds able to provide an effective control of PWN. 
Variability in essential oil composition and yield is known to occur, particularly due to physiological variation, environmental conditions, and geographic variation [18]. Despite differences due to tested concentration and/or plant part employed to obtain the oil, our results for oils with low effect are similar to those previously obtained for Cinnamomum camphora [19], Citrus aurantium [11], Eucalyptus globulus [11,19], Lippia citriodora [20] and Rosmarinus officinalis [11].

Also, results for effective oils match those previously obtained with $C$. citratus [11,19], results for $O$. vulgare are different from those previously obtained by Kong et al. [11]. Differences in the composition of the essential oils used may explain the different results obtained.

To our knowledge this is the first report of nematicide activity against PWN by $R$. graveolens. Among several properties, essential oils from $O$. vulgare, $R$. graveolens and $S$. montana showed antibacterial [21-23] and antifungal [24,25] capability.

\subsection{Chemical Profile of Essential Oils}

Of the 52 essential oils isolated and chemically characterized, only those of $S$. montana (Sm2 and Sm3) and $R$. graveolens ( $R g 1$ and $R g 2$ ) are detailed in Table 4, since they were the only ones that revealed $100 \%$ nematicide activity. Data for $O$. vulgare and $S m 1$ can be found elsewhere [8].

Table 4. Chemical composition of essential oils and volatiles of Portuguese plants resulting in $100 \%$ mortality of Bursaphelenchus xylophilus at $2 \mathrm{mg} / \mathrm{mL}$.

\begin{tabular}{|c|c|c|c|c|c|}
\hline \multirow[b]{2}{*}{ Compounds } & \multicolumn{3}{|c|}{ Lamiaceae } & \multirow{2}{*}{$\begin{array}{c}\text { Rutaceae } \\
\operatorname{Rg} 1 \\
\end{array}$} & \multirow[b]{2}{*}{$\operatorname{Rg} 2$} \\
\hline & RI $^{\mathrm{a}}$ & $S m 2$ & $S m 3$ & & \\
\hline 2-Methyloctane & 887 & - & - & $t^{b}$ & $\mathrm{t}$ \\
\hline Tricyclene & 921 & $\mathrm{t}$ & $\mathrm{t}$ & - & - \\
\hline$\alpha$-Thujene & 924 & 0.3 & 2.4 & - & - \\
\hline$\alpha$-Pinene & 930 & 1.6 & 2.3 & - & - \\
\hline Camphene & 938 & 1.6 & 0.1 & - & - \\
\hline 1-Octen-3-ol & 961 & $\mathrm{t}$ & $\mathrm{t}$ & - & - \\
\hline$\beta$-Pinene & 963 & 0.2 & 1.2 & - & - \\
\hline$n$-Octanal & 973 & - & - & $\mathrm{t}$ & $\mathrm{t}$ \\
\hline$\beta$-Myrcene & 975 & $\mathrm{t}$ & 2.7 & - & - \\
\hline$\alpha$-Phellandrene & 995 & $\mathrm{t}$ & 0.4 & - & - \\
\hline$\delta$-3-Carene & 1000 & $\mathrm{t}$ & 0.1 & - & - \\
\hline$\alpha$-Terpinene & 1002 & 0.3 & 4.1 & - & - \\
\hline p-Cymene & 1003 & 20.3 & 8.1 & - & - \\
\hline 1,8-Cineole & 1005 & $\mathrm{t}$ & $\mathrm{t}$ & - & - \\
\hline$\beta$-Phellandrene & 1005 & $\mathrm{t}$ & 0.1 & - & - \\
\hline Limonene & 1009 & 0.6 & 0.5 & - & - \\
\hline cis- $\beta$-Ocimene & 1017 & $\mathrm{t}$ & $\mathrm{t}$ & - & - \\
\hline$\gamma$-Terpinene & 1035 & 4.3 & 41.1 & - & - \\
\hline trans-Sabinene hydrate & 1037 & $\mathrm{t}$ & $\mathrm{t}$ & - & - \\
\hline 2-Nonanone & 1058 & - & - & $\mathrm{t}$ & $\mathrm{t}$ \\
\hline 2,5-Dimethyl styrene & 1059 & $\mathrm{t}$ & $\mathrm{t}$ & - & - \\
\hline Terpinolene & 1064 & 0.4 & $\mathrm{t}$ & - & - \\
\hline cis-Sabinene hydrate & 1066 & $\mathrm{t}$ & $\mathrm{t}$ & - & - \\
\hline
\end{tabular}


Table 4. Cont.

\begin{tabular}{|c|c|c|c|c|c|}
\hline \multirow[b]{2}{*}{ Compounds } & \multicolumn{3}{|c|}{ Lamiaceae } & \multirow{2}{*}{$\begin{array}{c}\text { Rutaceae } \\
\text { Rg1 }\end{array}$} & \multirow[b]{2}{*}{$\operatorname{Rg} 2$} \\
\hline & $\mathbf{R I}^{\mathrm{a}}$ & $\operatorname{Sm} 2$ & $S m 3$ & & \\
\hline$n$-Nonanal & 1073 & - & - & $\mathrm{t}$ & $\mathrm{t}$ \\
\hline Linalool & 1074 & $\mathrm{t}$ & $\mathrm{t}$ & - & - \\
\hline Geigerene isomer & 1116 & - & - & $\mathrm{t}$ & $\mathrm{t}$ \\
\hline Geigerene & 1121 & - & - & 0.5 & 0.1 \\
\hline Borneol & 1134 & 3.9 & 0.1 & - & - \\
\hline Terpinen-4-ol & 1148 & 2.3 & 0.2 & - & - \\
\hline$\alpha$-Terpineol & 1159 & $\mathrm{t}$ & $\mathrm{t}$ & - & - \\
\hline 2-Decanone & 1166 & - & - & $\mathrm{t}$ & $\mathrm{t}$ \\
\hline Carvacrol methyl ether & 1224 & 3.7 & $\mathrm{t}$ & - & - \\
\hline 2-Undecanone & 1275 & - & - & 94.4 & 92.8 \\
\hline Thymol & 1275 & 15.2 & $\mathrm{t}$ & - & - \\
\hline Carvacrol & 1286 & 40 & 35.3 & - & - \\
\hline$\beta$-Bourbonene & 1379 & $\mathrm{t}$ & $\mathrm{t}$ & - & - \\
\hline 2-Dodecanone ${ }^{c}$ & 1389 & - & - & $\mathrm{t}$ & $\mathrm{t}$ \\
\hline$\beta$-Caryophyllene & 1414 & 2.6 & 1.1 & - & - \\
\hline$\beta$-Copaene & 1426 & $\mathrm{t}$ & $\mathrm{t}$ & - & - \\
\hline Aromadendrene & 1428 & 0.3 & $\mathrm{t}$ & - & - \\
\hline$\alpha$-Humulene & 1447 & $\mathrm{t}$ & $\mathrm{t}$ & - & - \\
\hline 2-Tridecanone & 1479 & - & - & $\mathrm{t}$ & $\mathrm{t}$ \\
\hline$\beta$-Bisabolene & 1500 & 0.1 & $\mathrm{t}$ & - & - \\
\hline trans-Calamenene & 1505 & 0.1 & $\mathrm{t}$ & - & - \\
\hline$\delta$-Cadinene & 1505 & 0.1 & 0.1 & - & - \\
\hline$\beta$-Caryophyllene oxide & 1561 & 0.6 & $\mathrm{t}$ & - & - \\
\hline $\mathrm{UI} R g^{\mathrm{d}}$ & 1626 & - & - & 5.1 & 7.1 \\
\hline$\%$ of identification & & 98.6 & 99.9 & 94.9 & 92.9 \\
\hline \multicolumn{6}{|l|}{ Grouped components } \\
\hline Monoterpene hydrocarbons & & 29.6 & 63.1 & - & - \\
\hline Oxygen-containing monoterpenes & & 65.2 & 35.6 & - & - \\
\hline Sesquiterpene hydrocarbons & & 3.2 & 1.2 & - & - \\
\hline Oxygen-containing sesquiterpenes & & 0.6 & - & - & - \\
\hline C13 compounds & & - & - & 0.5 & 0.1 \\
\hline Others & & - & - & 94.4 & 92.8 \\
\hline
\end{tabular}

Satureja montana 2 (Sm2), S. montana 3 (Sm3), Ruta graveolens 1 (Rg1), R. graveolens 2 (Rg2).

${ }^{\mathrm{a}} \mathrm{RI}=$ Retention index relative to $\mathrm{C}_{8}-\mathrm{C}_{17} n$-alkanes on the DB1 column; ${ }^{\mathrm{b}} \mathrm{t}=\operatorname{trace}(<0.05 \%)$; ${ }^{\mathrm{c}}$ identification based on mass spectra only; ${ }^{\mathrm{d}}$ unidentified compound in $R$. graveolens essential oil; $m / z$ (rel. int.) $186[\mathrm{M}]^{+}$(3), 105 (12), 104 (62), 92 (18), 91 (68), 82 (12), 71 (37), 65 (17), 58 (17), $43(100)$.

O. vulgare (Lamiaceae) essential oil was dominated [8] by carvacrol $(36 \%), \gamma$-terpinene $(24 \%)$ and $p$-cymene (14\%). Carvacrol was also a major component $(35 \%-40 \%)$ of the essential oils of the three S. montana samples. Differences between essential oils of samples $S m 1$ [8] and $S m 3$ were quite few and their major constituents, by descending order, were $\gamma$-terpinene $(40 \%-41 \%)$, carvacrol and $p$-cymene $(7 \%-8 \%)$. However, differences were more considerable relative to sample $S m 2$, where 
$p$-cymene $(20 \%)$ was present in higher concentration and thymol $(15 \%)$ replaces $\gamma$-terpinene in the top three constituents.

Essential oils from fresh (1) and dried (2) R. graveolens (Rutaceae) were quite similar and characterized by few compounds. Only two compounds displayed major differences: 2-undecanone was more abundant in the fresh form, while an unidentified compound (UI $R g$, Table 4) was more abundant in the dry form.

The volatile profile of the two Lamiaceae species reported as having nematicide activity was in accordance with previous studies on $O$. vulgare [26,27] and S. montana [27,28]. For R. graveolens, previous studies also show 2-undecanone as the main component of the essential oil, although attaining only $34 \%-47 \%[29,30]$.

Chemical profiles of essential oils may provide useful guidelines to design efficient strategies to identify chemicals to be used for PWN control. In fact, essential oils of $R$. graveolens are almost completely constituted by 2 -undecanone ( $94.4 \%$ and $92.8 \%$ respectively), a compound absent from the essential oils not only of $S$. montana but also from the essential oils previously found to completely control PWN except in the case of Chamaespartium tridentatum were it was found in a relatively small amount [8]. Correspondences analysis of the percentage composition of the essential oils, which showed effective nematicide activity both in the present and in a previous study [8], supported the chemical differences between $R$. graveolens essential oils and all other tested essential oils (Figure 1).

The multidimensional pattern of chemicals composition strongly relies upon the amount of 2-undecanone and carvacrol, separating $R$. graveolens essential oils from the remaining oils by the amounts of those compounds. Hierarchical classification performed on the first two factors identifies two classes. One composed by $R$. graveolens essential oils and characterized by significantly high amounts of 2-undecanone and of an unidentified compound of $R$. graveolens, the other composed by all other essential oils and characterized by significantly low amounts of the same compounds.

Altogether these results strongly suggest that 2-undecanone could be the responsible for the effects of $R$. graveolens and simultaneously that one or more compounds not present in $R$. graveolens are also capable of completely control PWN.

Considering only the major constituents of essential oils other than those obtained from R. graveolens, $\mathrm{LC}_{50}$ in $\mathrm{PWN}$ treated with thymol and carvacrol was found to be $1.08 \mathrm{mg} / \mathrm{mL}$ and $1.23 \mathrm{mg} / \mathrm{mL}$ and higher than $20 \mathrm{mg} / \mathrm{mL}$ with $p$-cymene and $\gamma$-terpinene [31]. However, LC $_{100}$ associated with $R$. graveolens essential oils $(0.617 \pm 0.030 \mathrm{mg} / \mathrm{mL})$ is significantly lower $\left(p=3.5 \times 10^{-9}\right)$ than $\mathrm{LC}_{100}$ associated with all the remaining essential oils $(1.156 \pm 0.072 \mathrm{mg} / \mathrm{mL})$ and well below concentrations responsible for $\mathrm{LC}_{50}$ of single compounds. It is also well below concentrations responsible for $\mathrm{LC}_{50}$ of 2-undecanone applied alone to two species of root knot nematodes [32]. Therefore, 2-undecanone, the most likely responsible for the effects found with $R$. graveolens, is clearly a highly promising candidate for PWN control. To our knowledge, this is the first report of the nematicidal activity of this compound against PWN. 


\section{Experimental}

\subsection{Plant Material}

The aerial parts of several Portuguese flora species, from collective or individual samples, were collected from wild-growing plants in mainland Portugal and in the Madeira and Açores islands (Portugal). Plant material was stored at $-20{ }^{\circ} \mathrm{C}$ until extraction. Dried aerial parts from commercially available products sold in local herbal shops were also evaluated. In total, 52 essential oils from 29 taxa representing 12 families (Table 3) were evaluated for nematicide activity.

\subsection{Essential Oils and Volatiles Extraction}

Essential oils were isolated by hydrodistillation $(\mathrm{H})$ for $3 \mathrm{~h}$ using a Clevenger-type apparatus according to the European Pharmacopoeia method [33]. Volatiles were isolated by distillation-extraction (D-E) for $3 \mathrm{~h}$ using a Likens-Nickerson-type apparatus with $50 \mathrm{~mL}$ of distilled $n$-pentane (Riedel-de Haën, Sigma-Aldrich Laborchemikalien GmbH, Seelze, Germany) as the organic solvent (Table 3). Both isolation procedures were run at a distillation rate of $3 \mathrm{~mL} / \mathrm{min}$ and, on average, at least $100 \mathrm{~g}$ of each plant was extracted. The $\mathrm{D}-\mathrm{E}$ oils recovered in distilled $n$-pentane were concentrated at room temperature under reduced pressure on a rotary evaporator, collected in a vial, and concentrated to a minimum volume, again at room temperature, under nitrogen flux. Essential oils and volatiles were stored in the dark at $-20{ }^{\circ} \mathrm{C}$ until analysis.

\subsection{Rearing and Collection of Nematodes}

Wood chips from a maritime pine (Pinus pinaster Ainton) tree displaying wilt symptoms were collected in the Setúbal region, Portugal. Collected PWN were maintained in Petri dishes containing Botrytis cinerea cultured on malt extract agar. Prior to testing, cultured nematodes were separated from the agar medium for $48 \mathrm{~h}$ in a Baermann tray [34], placed in a new fungal mat, and left to multiply for one week at $25^{\circ} \mathrm{C}$ in the dark. Nematodes were separated from the culture medium as described above and counted under a binocular microscope Olympus SZX-12 (Olympus Corporation, Tokyo, Japan). A nematode suspension in distilled water was made by a series of dilutions, such that $100 \mu \mathrm{L}$ contained between 100 and 200 mixed-stage nematodes. The suspension was prepared immediately prior to use. The same PWN isolate was employed in a previous study [8].

\subsection{Bioassays}

Bioassays were performed in 96-well microtiter plates (Carl Roth $\mathrm{GmbH}+\mathrm{Co}$. KG, Karlsruhe, Germany). In each well, the nematode suspension $(99 \mu \mathrm{L})$ was added, followed by the essential oil solution $(1 \mu \mathrm{L})$ diluted in the assessed solvent. Plates were placed in a vortex apparatus at $500 \mathrm{rpm}$ for 2 min and stored at $25{ }^{\circ} \mathrm{C}$ in the dark. After $24 \mathrm{~h}$, dead and live nematodes were counted under a binocular microscope (Olympus SZX-12). Nematodes were considered dead if they did not move even when mechanically stimulated.

Comparison of Triton X-100 $(50 \mu \mathrm{g} / \mathrm{mL})$ and acetone (1\% v:v) effects on PWN mortality was done preparing 31 wells with the nematode suspension $(99 \mu \mathrm{L})$, followed by $1 \mu \mathrm{L}$ of Triton $\mathrm{X}-100$ 
(Scharlau Chemie, Barcelona, Spain) in distilled water solution $(5 \mathrm{~g} / \mathrm{mL})$ or $1 \mu \mathrm{L}$ of acetone (Carl Roth $\mathrm{GmbH}+$ Co. KG, Karlsruhe, Germany; 99.8\% purity) instead of Triton X-100; PWN mortality was recorded.

The same essential oils of $C$. citratus, $O$. vulgare and $S$. montana previously found [8] to result in more than $90 \%$ PWN mortality, at $2 \mathrm{mg} / \mathrm{mL}$ in Triton X-100 were used to compare essential oils effects on PWN mortality using Triton X-100 and acetone by testing oils at 2, 1, 0.5, 0.25 and $0.125 \mathrm{mg} / \mathrm{mL}$ using acetone as diluent. The higher concentration was prepared from the pure oil and acetone (99.8\% purity) which was included as control. The following dilutions series were prepared from the initial one. Each concentration was prepared once and five wells were used per essential oil and concentration. Mortality was recorded as described above and results compared with those previously found using Triton X-100.

Forty nine additional essential oils were tested at $2 \mathrm{mg} / \mathrm{mL}$ using acetone as dilution agent. Concentrations were prepared once and five wells were used per essential oil and concentration. Essential oils resulting in $100 \%$ mortality at $2 \mathrm{mg} / \mathrm{mL}$ in the five wells were further tested at $1,0.5$, 0.25 and $0.125 \mathrm{mg} / \mathrm{mL}$ as described above.

\subsection{Determination of Essential Oils Composition}

\subsubsection{Gas Chromatography (GC)}

Gas chromatographic analyses were performed using a Perkin Elmer Autosystem XL gas chromatograph (Perkin Elmer, Shelton, CT, USA) equipped with two flame ionization detectors (FIDs), a data handling system, and a vaporizing injector port into which two columns of different polarities were installed: a DB-1 fused-silica column $(30 \mathrm{~m} \times 0.25 \mathrm{~mm}$ i.d., film thickness $0.25 \mu \mathrm{m}$; J \& W Scientific Inc., Rancho Cordova, CA, USA) and a DB-17HT fused-silica column (30 $\mathrm{m} \times 0.25 \mathrm{~mm}$ i.d., film thickness $0.15 \mu \mathrm{m}$; J \& W Scientific Inc.). Oven temperature was programmed to increase from 45 to $175{ }^{\circ} \mathrm{C}$, in $3{ }^{\circ} \mathrm{C} / \mathrm{min}$ increments, then up to $300{ }^{\circ} \mathrm{C}$ in $15{ }^{\circ} \mathrm{C} / \mathrm{min}$ increments, and finally held isothermal for $10 \mathrm{~min}$. Gas chromatographic settings were as follows: injector and detectors temperatures, $280{ }^{\circ} \mathrm{C}$ and $300{ }^{\circ} \mathrm{C}$, respectively; carrier gas, hydrogen, adjusted to a linear velocity of $30 \mathrm{~cm} / \mathrm{s}$. The samples were injected using a split sampling technique, ratio 1:50. The volume of injection was $0.1 \mu \mathrm{L}$ of a pentane-oil solution (1:1). The percentage composition of the oils was computed by the normalization method from the GC peak areas, calculated as a mean value of two injections from each oil, without response factors.

\subsubsection{Gas Chromatography-Mass Spectrometry (GC-MS)}

The GC-MS unit consisted of a Perkin Elmer Autosystem XL gas chromatograph, equipped with DB-1 fused-silica column $(30 \mathrm{~m} \times 0.25 \mathrm{~mm}$ i.d., film thickness $0.25 \mu \mathrm{m}$; J \& W Scientific, Inc.) interfaced with Perkin-Elmer Turbomass mass spectrometer (software version 4.1, Perkin Elmer). GC-MS settings were as follows: injector and oven temperatures were as above; transfer line temperature, $280{ }^{\circ} \mathrm{C}$; ion source temperature, $220{ }^{\circ} \mathrm{C}$; carrier gas, helium, adjusted to a linear velocity of $30 \mathrm{~cm} / \mathrm{s}$; split ratio, 1:40; ionization energy, $70 \mathrm{eV}$; scan range, 40-300 u; scan time, $1 \mathrm{~s}$. The identity of the components was assigned by comparison of their retention indices relative to $\mathrm{C}_{8}-\mathrm{C}_{17} n$ 
alkane indices, and GC-MS spectra from a laboratory made library based upon the analyses of reference oils, laboratory-synthesized components, and commercial available standards.

\subsection{Data Analysis}

Effects of Triton X-100 and acetone on PWN mortality were compared by exact two-tailed Student $t$ test after checking for homocedasticity using the two-tailed $F$ distribution.

To account for the observed mortality in controls $\left(\mathrm{M}_{0}\right)$, mortality in treatments $\left(\mathrm{M}_{\mathrm{T}}\right)$ was corrected by the Schneider-Orelli's formula $\mathrm{M}_{\mathrm{C}}=\mathrm{M}_{\mathrm{T}}-\mathrm{M}_{0} / 100-\mathrm{M}_{0}$ [35] and expressed as percentage. Confidence intervals of $99 \%$ for $M_{C}$ were used to identify essential oils active against $P W N . M_{C}$ values at the tested essential oil concentrations using acetone were compared to $\mathrm{M}_{\mathrm{C}}$ values recorded at the same concentrations using Triton X-100 by exact or approximate two-tailed Student $t$ tests after checking for homocedasticity using the two-tailed $F$ distribution.

The relation between $\mathrm{M}_{\mathrm{C}}$ and essential oil concentration was investigated by fitting the Weibull function [17] by least squares nonlinear regression without replication using the Marquardt method [36].

The three parameter Weibull function is expressed as $\mathrm{M}_{\mathrm{C}}=1-\exp -\left\{[(\mathrm{X}-l) / k]^{c}\right\}$ where $\mathrm{M}_{\mathrm{C}}$ is the observed corrected mortality (in proportion) at essential oil concentration $X . l$ is a location parameter that for all practical purposes represents the minimum concentration of essential oil effective against PWN. $k$ is a scale parameter that represents the concentration at which the mortality is approximately $63 \%\left(\mathrm{LC}_{63}\right)$ when $l=0$. To control PWN effectively $\mathrm{LC}_{63}$ is clearly a less than desirable target and values of $\mathrm{LC}_{100}$ were calculated from fitted equations, since in the first situation the remaining population is able to quickly multiply and achieve the previous abundance. $c$ is a shape parameter that evaluates the symmetry of the distribution with $3.25 \leq c \leq 3.61$ showing symmetry and representing a good approximation to the normal distribution, $c<3.25$ positive, $c>3.61$ negative asymmetry [37,38].

Replicates were defined by their rank of corrected mortality and fitted equations were only accepted after a consistency check of parameter estimates and mortality predictions against original data. $l, c$ and $\mathrm{LC}_{100}$ values using acetone were compared to $l, c$ and $\mathrm{LC}_{100}$ values using Triton $\mathrm{X}-100$ by exact or approximate two-tailed Student $t$ tests after checking for homocedasticity using the two-tailed $F$ distribution. The effects of essential oils on $l, c$ and $\mathrm{LC}_{100}$ were compared using a least squares linear regression approach with dummy variables to prevent the occurrence of lack of "transitivity" [39,40]. Forward stepwise selection with replication was used and the candidate model included qualitative variables only, namely the species source of the essential oil (coded as 1, 0), with an experiment-wise type I error rate of 0.01 for coefficients calculated using Dunn-Šidák method [41,42]. A significant level of $P=0.01$ was used throughout. Results of bioassays are presented as means $\pm \mathrm{SE}$.

Essential oils chemical profiles resulting in $100 \% \mathrm{PWN}$ mortality at $2 \mathrm{mg} / \mathrm{mL}$ tested with acetone, Triton X-100 or both (Table 2 and [8]) were investigated by correspondences analysis followed by hierarchical classification using the generalized Ward criterion [43]. Chemicals occurring as trace were set as $0.01 \%$. Characterization of factors in correspondences analysis was done using absolute contributions. Classes were characterized by test values of variables with an experiment-wise type I error rate of 0.01 using Dunn-Šidák method [41,42]. LC $_{100}$ values for classes were compared by one-tailed approximate $t$ test after checking for homocedasticity using the two-tailed $F$ distribution. 


\section{Conclusions}

Acetone seems better suited for essential oil dilution in nematicidal bioassays than the commonly employed Triton X-100. To our knowledge this is the first report on the use of acetone as a way to dissolve essential oils in this kind of research. Essential oils from $O$. vulgare closely followed by those from $R$. graveolens appear as promising sources of phytochemicals worth being selected for bioassayguided search. This is also the first report of nematicide activity against PWN by $R$. graveolens.

In our opinion $\mathrm{LC}_{100}$ is preferable to $\mathrm{LC}_{50}$ as a way to assess the number of plants with nematicidal activity and strengthen the obtained results.

\section{Acknowledgements}

This work is funded by FEDER Funds through the Operational Programme for Competitiveness Factors - COMPETE and National Funds through FCT - Foundation for Science and Technology under the Strategic Project PEst-C/AGR/UI0115/2011 and research contract PTDC/AGR-CFL/117026/2010 and PTDC/AGR-CFL/120184/2010. J.M.S. Faria is grateful to FCT for PhD grant SFRH/BD/43738/2008.

\section{References}

1. Mota, M.; Vieira, P. Pine Wilt Disease: A Worldwide Threat to Forest Ecosystems; Springer: Heidelberg, Germany, 2008; pp. 1-3.

2. Fonseca, L.; Lopes, A.; Cardoso, J.; Pestana, M.; Abreu, F.; Nunes, N.; Mota, M.; Abrantes, I. The Pinewood Nematode, Bursaphelenchus xylophilus, in Madeira Island. Presented at the Abstracts of the 30th International Symposium of European Society of Nematologists, Vienna, Austria, 19-23 September 2010; p. 176.

3. Abelleira, A.; Picoaga, A.; Mansilla, J.P.; Aguin, O. Detection of Bursaphelenchus xylophilus, causal agent of pine wilt disease on Pinus pinaster in Northwestern Spain. Plant Dis. 2011, 95, 776.

4. Portaria N. 553-B/2008, Ministério da Agricultura, Desenvolvimento Rural e Pescas; Diário da República, Série 1, n. 123; Imprensa Nacional Casa da Moeda: Lisboa, Portugal, 27 June 2008; pp. 4000(16)-4000(19).

5. Alen, Y.; Nakajima, S.; Nitoda, T.; Baba, N.; Kanzaki, H.; Kawazu, K. Antinematodal activity of some tropical rainforest plants against the pinewood nematode, Bursaphelenchus xylophilus. Z. Naturforsch. C 2000, 55, 295-299.

6. Choi, I.H.; Shin, S.C.; Park, I.K. Nematicidal activity of onion (Allium cepa) oil and its components against the pine wood nematode (Bursaphelenchus xylophilus). J. Nematol. 2007, 9, 231-235.

7. Hong, L.; Li, G.; Zhou, W.; Wang, X.; Zhang, K. Screening and isolation of a nematicidal sesquiterpene from Magnolia grandiflora L. Pest Manag. Sci. 2007, 63, 301-305.

8. Barbosa, P.; Lima, A.S.; Vieira, P.; Dias, L.S.; Tinoco, M.T.; Barroso, J.G.; Pedro, L.G.; Figueiredo, A.C.; Mota, M. Nematicidal activity of essential oils and volatiles derived from Portuguese aromatic flora against the pinewood nematode, Bursaphelenchus xylophilus. J. Nematol. 2010, 42, 8-16. 
9. UNEP Chemicals (United Nations Environment Programme) (2007). Screening Information Datasets for High Volume Chemicals. Available online: http://www.chem.unep.ch/irptc/sids/ OECDSIDS/sidspub.html (accessed on 1 October 2012).

10. Ademola, I.O.; Eloff, J.N. Ovicidal and larvicidal activity of Cassia alata leaf acetone extract and fractions on Haemonchus contortus: In vitro studies. Pharm. Biol. 2011, 49, 539-544.

11. Kong, J.O.; Lee, S.M.; Moon, Y.S.; Lee, S.G.; Ahn, Y.J. Nematicidal activity of plant essential oils against Bursaphelenchus xylophilus (Nematoda: Aphelenchoididae). J. Asia-Pacific Entomol. 2006, 9, 173-178.

12. Park, I.K.; Kim, J.; Lee, S.G.; Shin, S.C. Nematicidal activity of plant essential oils and components from Ajowan (Trachyspermum ammi), Allspice (Pimenta dioica) and Litsea (Litsea cubeba) essential oils against pine wood nematode (Bursaphelenchus xylophilus). J. Nematol. 2007, 39, 275-279.

13. Elbadri, G.A.; Lee, D.W.; Park, J.C.; Yu, W.B.; Choo, H.Y.; Lee, S.M.; Lim, T.H. Nematocidal screening of essential oils and herbal extracts against Bursaphelenchus xylophilus. Plant Pathol. J. 2008, 24, 178-182.

14. Kim, J.; Seo, S.M.; Lee, S.G.; Shin, S.C.; Park, I.K. Nematicidal Activity of Plant Essential oils and components from Coriander (Coriandrum sativum), Oriental Sweetgum (Liquidambar orientalis), and Valerian (Valeriana wallichii) essential oils against Pine Wood Nematode (Bursaphelenchus xylophilus). J. Agric. Food Chem. 2008, 56, 7316-7320.

15. Limpert, E.; Stahel, W.A.; Abbt, M. Log-normal distributions across the sciences: Keys and clues. BioScience 2001, 51, 341-352.

16. Takai, K.; Soejima, T.; Suzuki, T.; Kawazu, K. Development of a water-soluble preparation of emamectin benzoate and its preventative effect against the wilting of pot-grown pine trees inoculated with the pine wood nematode, Bursaphelenchus xylophilus. Pest Manag. Sci. 2001, 57, 463-466.

17. Weibull, W. A statistical distribution function of wide applicability. J. Appl. Mech. 1951, 18, 293-297.

18. Figueiredo, A.C.; Barroso, J.G.; Pedro, L.G.; Salgueiro, L.; Miguel, M.G.; Faleiro, M.L. Portuguese Thymbra and Thymus species volatiles: Chemical composition and biological activities. Curr. Pharm. Des. 2008, 14, 3120-3140.

19. Park, I.K.; Park, J.Y.; Kim, K.H.; Choi, K.S.; Choi, I.H.; Kim, C.S.; Shin, S.C. Nematicidal activity of plant essential oils and components from garlic (Allium sativum) and cinnamon (Cinnamomum verum) oils against the pine wood nematode (Bursaphelenchus xylophilus). Nematology 2005, 7, 767-774.

20. Kim, J.; Seo, S.M.; Park, I.K. Nematicidal activity of plant essential oils and components from Gaultheria fragrantissima and Zanthoxylum alatum against the pine wood nematode, Bursaphelenchus xylophilus. Nematology 2011, 13, 87-93.

21. Carramiñana, J.J.; Rota, C.; Burillo, J.; Herrera, A. Antibacterial efficiency of Spanish Satureja montana essential oil against Listeria monocytogenes among natural flora in minced pork. J. Food Protect. 2008, 71, 502-508.

22. Nogueira, J.C.; Diniz, M.F.; Lima, E.O. In vitro antimicrobial activity of plants in Acute Otitis Externa. Braz. J. Otorhinolaryn. 2008, 74, 118-124. 
23. Soković, M.; Glamočlija, J.; Marin, P.D.; Brkić, D.; van Griensven, L.J. Antibacterial effects of the essential oils of commonly consumed medicinal herbs using an in vitro model. Molecules 2010, 15, 7532-7546.

24. Giordani, R.; Regli, P.; Kaloustian, J.; Mikaïl, C.; Abou, L.; Portugal, H. Antifungal effect of various essential oils against Candida albicans. Potentiation of antifungal action of amphotericin B by essential oil from Thymus vulgaris. Phytother. Res. 2004, 18, 990-995.

25. Lazar-Baker, E.E.; Hetherington, S.D.; Ku, V.V.; Newman, S.M. Evaluation of commercial essential oil samples on the growth of postharvest pathogen Monilinia fructicola (G. Winter) Honey. Lett. Appl. Microbiol. 2011, 52, 227-232.

26. Faleiro, L.; Miguel, G.; Gomes, S.; Costa, L.; Venâncio, F.; Teixeira, A.; Figueiredo, A.C.; Barroso, J.G.; Pedro, L.G. Antibacterial and antioxidant activities of essential oils isolated from Thymbra capitata L. (Cav.) and Origanum vulgare L. J. Agric. Food Chem. 2005, 53, 8162-8168.

27. Prieto, M.; Iacopini, P.; Cioni, P.; Chericoni, S. In vitro activity of the essential oils of Origanum vulgare, Satureja montana and their main constituents in peroxynitrite-induced oxidative processes. Food Chem. 2007, 104, 889-895.

28. Grosso, C.; Oliveira, A.C.; Mainar, A.M.; Urieta, J.S.; Barroso, J.G.; Palavra, A.M.F. Antioxidant activities of the supercritical and conventional Satureja montana extracts. J. Food Sci. 2009, 74, 713-717.

29. Feo, V.; Simone, F.; Senatore, F. Potential allelochemicals from the essential oil of Ruta graveolens. Phytochemistry 2002, 61, 573-578.

30. Soleimani, M.; Azar, P.A.; Saber-Tehranil, M.; Rustaiyan, A. Volatile composition of Ruta graveolens L. of North of Iran. World Appl. Sci. J. 2009, 7, 124-126.

31. Kong, J.O.; Park, I.K.; Choi, K.S.; Shin, S.C.; Ahn, Y.J. Nematicidal and propagation activities of thyme red and white oil compounds toward Bursaphelenchus xylophilus (Nematode: Parasitaphelenchidae). J. Nematol. 2007, 39, 237-242.

32. Ntalli, N.G.; Manconi, F.; Leonti, M.; Maxia, A.; Caboni, P. Aliphatic ketones from Ruta chalepensis (Rutaceae) induce paralysis on root knot nematodes. J. Agric. Food Chem. 2011, 59, 7098-7103.

33. Council of Europe. European Pharmacopoeia, 7th ed.; European Directorate for the Quality of Medicines: Strasbourg, France, 2010; p. 241.

34. Viglierchio, D.R.; Schmitt, R.V. On the methodology of nematode extraction from field samples: Baermann funnel modifications. J. Nematol. 1983, 15, 438-444.

35. Püntener, W. Manual for Field Trials in Plant Protection, 2nd ed.; Ciba-Geigy: Basle, Switzerland, 1981.

36. Marquardt, D.W. An algorithm for least-squares estimation of nonlinear parameters. J. Soc. Ind. Appl. Math. 1953, 11, 431-441.

37. Dubey, S.D. Normal and Weibull distributions. Nav. Res. Logist. Q. 1967, 14, 69-79.

38. Bonner, F.T.; Dell, T.R. The Weibull function: A new method of comparing seed vigor. J. Seed Technol. 1976, 1, 96-103.

39. Chew, V. Comparing treatment means: a compendium. HortScience 1976, 11, 348-357.

40. Penas, A.C.; Dias, L.S.; Mota, M.M. Precision and selection of extraction methods of aphelenchid nematodes from maritime pine wood, Pinus pinaster L. J. Nematol. 2002, 34, 62-65. 
41. Ury, H.K. A comparison of four procedures for multiple comparisons among means (pairwise contrasts) for arbitrary simple sizes. Technometrics 1976, 18, 89-97.

42. Sokal, R.R.; Rohlf, F.J. Biometry. The Principles and Practice of Statistics in Biological Research, 3rd ed.; Freeman: New York, NY, USA, 1995; p. 239.

43. Lebart, L.; Morineau, A.; Piron, M. Statistique Exploratoire Multidimensionelle, 3éme ed., Dunod: Paris, France, 2000; pp. 170-171.

Sample Availability: No pure compounds were used. Samples of the tested essential oils are available from the authors.

(C) 2012 by the authors; licensee MDPI, Basel, Switzerland. This article is an open access article distributed under the terms and conditions of the Creative Commons Attribution license (http://creativecommons.org/licenses/by/3.0/). 